Proceedings of the $\mathbf{2}^{\text {nd }}$ ICEENG Conference, 23-25 Nov. 1999

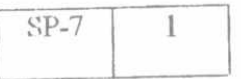

Military Technical College
Kobry Elkobbah,
Cairo, Egypt

$2^{\text {nd }}$ International Conference on Electrical Engineering ICEENG 99

\title{
BAUD DURATION ESTIMATION USING FAST COMPUTEDSPECTRAL CORRELATION FUNCTION OF M-ARY PSK SIGNALS
}

\author{
E. I. Eweda*, K. El-barbary", E. E. Azzouz", A. Al-Makhlouf"
}

\section{ABSTRACT}

This paper is concerned with the baud duration estimation of M-ary PSK signals with $M=2$, and 4 . The baud duration is one of the important parameters to intercept, and recognize digitally modulated signals as well as to determine the optimum jamming for them. The proposed method for baud duration estimation is based on the computation of the spectral correlation function of a signal. The proposed method for baud duration estimation provides reliable and accurate results at weak SNR. It is found that, a good estimation for baud duration of M-ary PSK signals at the SNR of $-5 \mathrm{~dB}$ is available. Also, in this paper a new method for fast computation of the spectral correlation function is provided.

\section{KEYWORDS}

Cyclostationary, cyclic autocorrelation, spectral correlation function, baud duration estimation,

\footnotetext{
"Egyption Armed Forces.

"syrian Armed Forces.
} 
Proceedings of the $\mathbf{2}^{\text {nd }}$ ICEENG Conference, 23-25 Nov. 1999

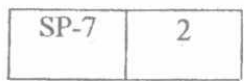

\section{I- INTRODUCTION}

Interception of communication signals is attempted for a variety of reasons including reconnaissance, surveillance, position fixing, identification, and communications jamming. For example, an aircraft might attempt to intercept the communications between a submarine or ship and a satellite, or a satellite might attempt to intercept ground-to-ground communications. Typically, the interceptor has knowledge of no more than the communicator frequency band and modulation format. In the past, most appropriate approaches for signal interception were based on radiometry[1]. However, it is commonly recognized that such radiometric methods can be highly susceptible to unknown and changing noise levels and interference activity. There have been many proposals for counteracting such complications, including various approaches to adjust or adapt threshold levels, adaptive filtering, and directional nulling of interfering signals. But these problems remain as the most serious impediment to signal interception tasks[1].

The radiometric approach for interception is based on the use of stationary random processes as models for signals to be intercepted. However, it is found that, for the purposes of signal interception, the signal of interest is more appropriately modeled as a cyclostationary random process. That is, a random process whose probabilistic or statistical parameters vary periodically with time[2]. The message contained in the modulated signal is unknown, and is usually modeled as a stationary random process. This stationarity coupled with the periodicity of sine wave carrier, pulse trains, repeating spreading codes,...etc., results in a cyclostationary model for the modulated signal. These cyclostationary signals typically do not exhibit spectral lines because the spectral lines of the unmodulated carrier and/or pulse trains are spread out over relatively broad bands by the stationary random modulation.

Before proceeding to the technical part of this paper, let us consider the signal interception problem and the weaknesses and strengths of radiometry and cyclic-feature detection in a little more detail. There is a clear trend towards increased use of systems employing sophisticated signal formats such as directsequence and frequency-hopped spread spectrum modulation. These techniques are to aid communication in this environment and to protect the communication system against interception. In these applications, it is unlikely that conventional interceptor will be able to perform required signal interception or subsequent analysis tasks. In addition, the modulation format of the signal of interest can make it indistinguishable from the background noise. The presence of several identically distributed spectrally superimposed signals will confuse most energy interception schemes, preventing the interceptor from determining any more than knowledge that signals are present in the environment. In particular, energy detection schemes are inherently unable to measure or exploit timing or phasing properties (carrier frequency, chip, or baud timing) of the signals of interest or interferences because these energy detectors usually cannot exploit the cyclostationary, of the signal characteristics.

There are some important advantages of cyclic spectral analysis over energy detection techniques[1]. One of them is its discriminatory capability. Signal features are discretely distributed in cyclic spectrum, even if the signal has continuous distribution in the power spectrum. Thus, signals with overlapping feature in the power spectrum can have non-overlapping features in the cyclic spectrum. Background noise, for instance, has no features at nonzero cyclic frequencies; i.e. analyzing the cyclic spectrum at a nonzero cyclic frequency where a 
signal-of-interest is expected to appear without any component due to the background noise. Another advantage of cyclic spectral analysis is that the cyclic spectrum is a richer domain for signal analysis than the conventional power spectrum. In addition to the signal separation provided by the cyclic spectrum magnitude, sine-wave carrier, pulse-train frequency, and phase parameters can also be measured from the cyclic spectrum magnitude and phase.

In section II, theoretical background and basic terminologies for spectral correlation function are presented. In section III, baud duration estimation algorithms are discussed and are provided in a new technique using spectral correlation function. In section IV, computer simulations for measuring spectral correlation function for BPSK and QPSK signals are given. Also, the performance evaluations for baud duration estimation are provided. Finally, the paper is concluded in section $\mathrm{V}$.

\section{II- THEORETICAL BACKGROUND AND BASIC TERMINOLOGIES}

For the purposes of signal interception, the signal of interest is most appropriately modeled as a cyclostationary random process, whose probabilistic or statistical parameters vary periodically with time, reflecting the characteristic property of regenerative periodicity. If there is more than one source of periodicity and the periods are not all commensurable, then the process is called almost cyclostationary since its parameters are almost periodic functions of time.

A zero-mean process $x(t)$ is said to be cyclostationary in wide sense if its autocorrelation is a periodic function of time, i.e.

$$
R_{x}(t+\tau / 2, t-\tau / 2)=R_{x}\left(t+T_{0}+\tau / 2, t+T_{0}-\tau / 2\right)
$$

for some period $T_{0} \neq 0$ where

$$
R_{x}(t+\tau / 2, t-\tau / 2) \stackrel{\Delta}{=} E\left\{x(t+\tau / 2) x^{*}(t-\tau / 2)\right\},
$$

and $E\{\}$ denotes the mathematical expectation operation. Since $R_{x}$ is periodic, it admits a Fourier series representation,

$$
R_{x}(t+\tau / 2, t-\tau / 2)=\sum_{\alpha} R_{x}^{\alpha}(\tau) e^{i 2 \pi \alpha t}
$$

where the sum over $\alpha$ includes all multiples of the reciprocal of the fundamental period $T_{0}$ (such as carrier frequency, baud rate, chip rate, hop rate, and their sums and differences). The Fourier coefficients $R_{x}^{\alpha}(\tau)$, which depend on the lag parameter $\mathrm{T}$, are given by

$$
R_{x}^{\alpha}(\tau)=\lim _{z \rightarrow \infty} \frac{1}{z} \int_{z / 2}^{z / 2} R_{x}(t+\tau / 2, t-\tau / 2) e^{-i 2 \pi \alpha t} d t .
$$

If there is only one period, say $T$, then $Z$ can be chosen equal to $T$, and the limit in (4) can be omitted. If $x(t)$ is a cycloergodic process[2,7], which will be the case if an appropriate model is used, then after substitution of (2) into (4), the expectation operator can be omitted to obtain

$$
\hat{R}_{x}^{\alpha}(\tau)=\lim _{z \rightarrow \infty} \frac{1}{z} \int_{z / 2}^{/ 2} x(t+\tau / 2) x(t-\tau / 2) e^{-i 2 \pi \alpha t} d t .
$$

When (5) is used in place of (4), the limit $Z \rightarrow \infty$ cannot be omitted if there is only one source of periodicity. We shall focus our analysis on the class of time-series for which the function $\hat{R}_{x}^{\alpha}$ exists and non identically zero for some non zero values of $\alpha$. Also, in order to avoid anomalous time-series, it is assumed that $\hat{R}_{x}^{\alpha}(\tau)$ is a continuous function of $\tau$. For $\alpha=0, \hat{R}_{x}^{\alpha}$ is the conventional limit autocorrelation, 
denoted by $\hat{R}_{x}$, which plays a fundamental role in the theory of conventional spectral analysis[2,4]. For $\alpha \neq 0, \hat{R}_{x}^{\alpha}$ is a generalization of the limit autocorrelation that incorporates a cyclic (sinusoidal) weighting function, and $\hat{R}_{x}^{\alpha}$ shall therefore be referred to the limit cyclic autocorrelation or sometimes abbreviated to cyclic autocorrelation. Whereas $\hat{R}_{x}^{\alpha}(\tau)$, for fixed $\tau$, is the constant (dc) component of the time-series

$$
z(t) \stackrel{\Delta}{=} x(t+\tau / 2) x(t-\tau / 2)
$$

$\hat{R}_{x}^{\alpha}(\tau)$ is the sine-wave (ac) component, with frequency $\alpha$, of the time-series $z(t)$. The set $\left\{\alpha: R_{x}^{\alpha}(\tau) \neq 0\right\}$ is refered to the set of cyclic frequencies. By analogy with the terminology for conventional autocorrelation $(\alpha=0$ in (5)), the Fourier transform of the cyclic autocorrelation, $S_{x}^{\alpha}(f)$, is given by,

$$
S_{x}^{\alpha}(f) \stackrel{\Delta}{\Delta} \int_{-\infty}^{+\infty} R_{x}^{\alpha}(\tau) e^{-i 2 \pi f \tau} d \tau,
$$

and it is called the cyclic spectral density function. For $\alpha=0$ it reduces to the conventional power spectral density function. Specifically, it is shown in [2]-[5] that

$$
S_{x}^{\alpha}(f)=\lim _{\Delta f \rightarrow 0} \lim _{\Delta t \rightarrow \infty} \frac{1}{\Delta t} \int_{-\Delta t / 2}^{\Delta t / 2} \Delta f X_{1 / \Delta f}(t, f+\alpha / 2) . X_{1 / \Delta f}^{*}(t, f-\alpha / 2) d t
$$

where $X_{1 / \Delta f}(t, f)$ is the complex envelope of the narrow-band spectral component with center frequency $f$ and bandwidth of order $\Delta f$;i.e.

$$
X_{1 / \Delta f}(t, f)=\int_{t-1 / 2 \Delta f}^{t+1 / 2 \Delta f} x(u) e^{-i 2 \pi f u} d u \text {. }
$$

By noting that $z \equiv \Delta t$ and $T \equiv 1 / \Delta f$, then $S_{x}^{\alpha}(f)$ is also called the spectral correlation function. Equation (8) represents the limit, as spectral resolution becomes infinitesimal $(\Delta f \rightarrow 0)$, and the limit $(\Delta t \rightarrow \infty)$ temporal correlation of the two spectral components at frequencies $f+\alpha / 2$ and $f-\alpha / 2$. Since the frequencies of the correlated spectral components are $f+\alpha / 2$ and $f-\alpha / 2$, the cycle frequency $\alpha$ is also called the frequency separation.

Now we provide several alternatives but equivalent definitions of the spectral correlation function. In addition to the two definitions (7) and (8), it can be shown that $S_{x}^{\alpha}(f)$ is given by the following limit of spectrally smoothed products of the spectral components of $x(t)$.

$$
S_{x}^{\alpha}(f)=\lim _{\Delta f \rightarrow 0} \lim _{\Delta t \rightarrow \infty} \frac{1}{\Delta f} \int_{f-\Delta f / 2}^{f+\Delta f / 2} \frac{1}{\Delta t} X_{\Delta t}(t, f+\alpha / 2) . X_{\Delta t}^{*}(t, f-\alpha / 2) d t,
$$

where $X_{\Delta t}(t, f)$ is defined by (9) if $1 / \Delta f$ is replaced by $\Delta t$. It can be shown that (5) is expressed by

$$
R_{x}^{\alpha}(\tau)=\lim _{\Delta t \rightarrow \infty} \frac{1}{\Delta t} \int_{\Delta t / 2}^{\Delta t / 2} u^{\alpha}(t+\tau / 2) v^{\alpha^{*}}(t-\tau / 2) d t
$$

where

$$
u^{\alpha}(t)=x(t) e^{-i \pi \alpha t} \text {, and } v^{\alpha}(t)=x(t) e^{+i \pi \alpha t}
$$

Thus, $R_{x}^{\alpha}(\tau)$ is the cross-correlation between $u^{\alpha}(t)$ and $v^{\alpha}(t)$, and $S_{x}^{\alpha}(f)$ is the Fourier transform of (11) as defined by (7). For convenience of the sequel, the integrand in definitions (8) and (10) is denoted by 


$$
S_{x T}^{\alpha}(t, f)=\frac{1}{T} X_{T}(t, f+\alpha / 2) X_{T}^{*}(t, f-\alpha / 2),
$$

and is called the cyclic periodogram. The symbol $T$ is used as a dummy parameter, which may be either $T \equiv 1 / \Delta f$ as in (8) or $T=\Delta t$ as in (10).

From the previous discussion we can conclude a new method to compute the spectral correlation function. First, two new signals from the original signal $x(t)$ are formed such as

$$
x_{1}^{\alpha}(t)=x(t) e^{i \pi \alpha t}, \quad \text { and } \quad x_{2}^{\alpha}(t)=x(t) e^{-i \pi \alpha t}
$$

Instead of computing the cross-correlation between $x_{1}^{\alpha}(t)$ and $x_{2}^{\alpha}(t)$ to find $S_{x}^{\alpha}(f)$, the Fourier transform is applied to $x_{1}^{\alpha}(t)$ and $x_{2}^{\alpha}(t)$ directly as

$$
X_{1}^{\alpha}(f)=F \cdot T \cdot\left\{x_{1}^{\alpha}(t)\right\}, \text { and } X_{2}^{\alpha}(f)=F \cdot T \cdot\left\{x_{2}^{\alpha}(t)\right\}
$$

and then the spectral correlation function $S_{x}^{\alpha}(f)$ is computed as

$$
S_{x}^{\alpha}(f)=X_{1}^{\alpha}(f) X_{2}^{\alpha}(f) \text {. }
$$

Computer simulations shows that computing the spectral correlation function using the Fourier transform method, defined by equations (14)-(16), is faster than the direct time domain method.

\section{III- BAUD DURATION ESTIMATION}

The spectral correlation concept has associated fundamental properties that are of significant practical value: 1) different types of modulated signals (such as BPSK, and QPSK) that have identical power spectral density functions can have highly distinct spectral correlation functions, 2) stationary noise and interference exhibit no spectral correlation (the spectral correlation function is identically zero), 3) the spectral correlation function contains phase and frequency information related to timing parameters in the modulated signals, and 4) the existence of spectral correlation function in a signal means that some spectral components can be estimated using other spectral components of the signal. These properties can be exploited for detection, classification, parameter estimation, and extraction of signals buried noise and interference. Although the spectral correlation function is a secondorder (quadratic) statistic like the power spectral density function, their properties enable us to accomplish tasks that are impossible to accomplish by the power spectral density function[3]. This includes synchronization[8], noise and interference rejection for signal extraction and detection[3].

As an example, the spectral correlation function of BPSK signal is given as below. M-ary PSK signals can be modeled by

$$
s(t)=\sum_{n=-\infty}^{\infty} q\left(t-n T_{b}-t_{0}\right) \cos \left(2 \pi f_{0} t+\theta_{n}+\phi_{0}\right)
$$


where $T_{b}$ is the chip interval, $\left\{\theta_{n}\right\}$, is an independent sequence of equiprobable values. In BPSK $\left\{\theta_{n}=0\right.$ and $\left.\pi\right\}$ and for QPSK $\left\{\theta_{n}=0, \pi / 2, \pi\right.$, and $\left.3 \pi / 2\right\}$. In this case the spectral correlation function of BPSK is given by $[2,3]$

$$
S_{s}^{\alpha}(f)=\left\{\begin{array}{lc}
\frac{1}{4 T_{c}} Q\left(f+\alpha / 2 \mp f_{0}\right) Q^{*}\left(f-\alpha / 2 \pm f_{0}\right) e^{-i\left[2 \pi\left(\alpha \mp 2 f_{0}\right) t_{0} \pm 2 \phi_{0}\right]}, & \alpha=\mp 2 f_{0}+\frac{k}{T_{b}} \\
\frac{1}{4 T_{c}}\left\{\begin{array}{l}
Q\left(f+\alpha / 2+f_{0}\right) Q^{*}\left(f-\alpha / 2+f_{0}\right) \\
+Q\left(f+\alpha / 2-f_{0}\right) Q^{*}\left(f-\alpha / 2-f_{0}\right)
\end{array}\right\} e^{-i 2 \pi \alpha t_{0}}, & \alpha=\frac{k}{T_{b}},
\end{array}\right.
$$

for all integers $k$, where $Q(f)$ is the Fourier transform of the pulse train, $q(t)$, and it is given by

$$
Q(f)=\frac{\sin \left(\pi f T_{b}\right)}{\pi f},
$$

Also, QPSK signal has spectral correlation function equal to that of the BPSK signal for $\alpha=k / T_{b}$ but not exists for $\alpha= \pm 2 f_{c}+k / T_{b}$.

It is well known that the BPSK and QPSK signals have identical power spectral densities[10]. On the other hand, their spectral correlation functions are different as shown in Fig. 1; specially at $\alpha \neq 0$. Thus, the spectral correlation function can be used to discriminate between BPSK and QPSK signals.

The baud duration estimation is very important for automatic signal classification as well as for signal recovery and information extraction. Generally, any baud duration estimation method used for a particular application depends both on the amount of the prior information available about possible baud durations and the constraints on processing time and estimation accuracy. Based on the amount of the prior information available, there are three possible situations. These are: 1) systems with no prior information, 2)systems with a defined range of parameters values, and 3) systems with a defined list of parameters values.

Baud duration estimation was studied in details in [11]-[13] and there were many methods to estimate the baud duration. It is worth noting that these methods depend on extracting the symbol sequence. Therefore, these methods are affected, deeply, by the noise. Results in [11]-[13] show that these methods are good at high SNR values, whereas, these methods fall when the SNR is less than $10 \mathrm{~dB}$.

In this paper, a new method for baud duration estimation is provided. This method depends on computing the spectral correlation function of an intercepted signal. By observing the spectral correlation function at $\alpha=k / T_{b}$ for all integers $k$, where $T_{b}$ is the baud duration of the symbol sequence for any one of considered signals, the baud duration can be estimated as the distance between the features at $\alpha=0$ and $\alpha=k / T_{b}$. As a corollary, the baud duration can be estimated by estimating the spectral correlation function of the intercepted signal.

\section{IV-COMPUTER SIMULATIONS AND PERFORMANCE EVALUATIONS}

Two cyclostationary signals are simulated. These signals are a binaryphase-shift-keyed (BPSK) carrier, with a binary phase-modulating (data) sequence, and a quaternary-phase-shift-keyed (QPSK) carrier with a quaternary phasemodulating sequence. For these two signals, the frame length is of 32768 samples, 
the carrier frequency, $f_{c}=2 f_{b}$, and the sampling rate, $f_{s}=4 f_{c}$, where $f_{b}$ is the bit rate. The computation of the spectral correlation function is performed by any of the two methods discussed in section II. First case depends on equations (5) and (7). The second case is by using the method described by equations (14)-(16). These simulations are used to find the spectral correlation functions of BPSK and QPSK signals. It is found that, computation of spectral correlation function using the second method is faster than the case of using equations (5) and (7). Fig.2 shows the spectral correlation functions of simulated one frame of both BPSK and QPSK signals. These functions are graphed as the height of a surface above the bifrequency $(f, \alpha)$ plane. To illustrate the peak locations on the bi-frequency $(f, \alpha)$ plane, contours are plotted as in the Fig.3. As shown from Figs.2 and 3, these signals have the same power spectral densities, whereas they have highly distinct spectral correlation function at $\alpha \neq 0$. This corollary is very useful for signal classification, that is, it is possible to distinguish among signals even if they have the same power spectral densities. Depending on the analysis of the properties of spectral correlation function, the distance between the first two peaks (at $\alpha=0$ and the second peak) is measured. This distance will be the symbol rate and consequently the baud duration is its reciprocal.

Baud duration estimation was tested for BPSK and QPSK signals corrupted with additive white Gaussian noise (WGN). This estimation task is done at different signal-to-noise ratio (SNR) values as shown in the table (1). As known white Gaussian noise has no features in the spectral correlation function at $\alpha \neq 0$ [1]. Thus, the effect of white Gaussian noise on a cyclostationary signal at $\alpha \neq 0$ will vanishes and the signal features will appear obviously. Thus, we will note that the baud duration estimation is validated successfully at low SNR. Table (1) presents the probability of correct estimation of baud duration of BPSK and QPSK signals. It is clear that, at $S N R=-5 \mathrm{~dB}$ and estimation error $\pm 2 \%$, the success rate is $100 \%$ for BPSK signal, while it is $90 \%$ for QPSK signal. These results are derived from 10 frames for each modulation type of interest.

Comparing the developed method with those presented in [11]-[13], we find the following points.

1) The developed method, based on the spectral correlation function, deals with RF signal itself not with the extracted symbol sequence as in [11]-[13].

2) The methods in [11]-[13] assume there is a prior knowledge about the range of values for baud duration. On the other hand, the developed method deals with no prior information systems.

3) The developed method provides reliable and accurate results at weak SNR. It is found that, good estimation for baud duration of M-ary PSK signals at the SNR of $-5 \mathrm{~dB}$ is available. On the other hand, the methods in [11]-[13] fall when the SNR is less than $10 \mathrm{~dB}$.

\section{V- CONCLUSIONS}

This paper is concerned with the baud duration estimation of M-ary PSK signals with $M=2$, and 4 . A new method for baud duration estimation is provided. This new method is based on the computation of the spectral correlation function of a signal. Also, in this paper a new method for fast computation of the spectral correlation function is provided. Comparing the developed method for baud duration estimation with those presented in [11]-[13], we find that the developed method, 
based on the spectral correlation function, deals with RF signal itself not with the extracted symbol sequence as in [11]-[13]. Methods in [11]-[13] assume there is a prior knowledge about the range of values for baud duration. On the other hand, the developed method deals with no prior information systems. The developed method provides reliable and accurate results at weak SNR. It is found that, good estimation for baud duration of M-ary PSK signals at the SNR of $-5 \mathrm{~dB}$ is available. On the other hand, the methods in [11]-[13] fail when the SNR is less than $10 \mathrm{~dB}$.

The current trend is the using of the spectral correlation function in the signal detection task.

\section{REFERENCES}

[1] W. A. Gardner," Signal interception: A unifying theoretical framework for feature detection," IEEE Trans. Commun. Vol. 36, pp. 897-906, Aug. 1988.

[2] W. A. Gardner, Introduction to Random Processes with Applications to Signals and Systems. New York; McGraw-Hall, 1989, $2^{\text {nd }}$ ed.

[3] W. A. Gardner, Statistical Spectral Analysis: A Non-probabilistic Theory. Englewood Cliffs, NJ: Prentice-Hall, 1987

[4] W. A. Gardner," The spectral correlation theory of cyclostationary timeseries," Signal Processing, vol. 11, pp. 13-36, July 1986.

[5] W. A. Gardner," Spectral correlation of modulated signals. Part IAnalog modulation," IEEE Trans. Commun., vol. COM-35, pp. 584-594, June 1987.

[6] W. A. Gardner," Characterization of cyclostationary random signal processes," IEEE Trans. Inform. Theory, vol. IT-21, pp. 4-14, Jan. 1975.

[7] R. A. Boyles and W. A. Gardner," Cycloergodic properties of discreteparameters non-stationary stochastic processes," IEEE Trans. Inform. Theory, vol. IT-29, pp. 105-114, Jan. 1983.

[8] W. A. Gardner," The role of spectral correlation in design and performance analysis of synchronizers," IEEE Trans. Commun., vol. COM-34, pp. 1089-1095, Nov. 1986.

[9] W. A. Gardner," Structural characterization of locally optimum detectors in terms of locally optimum estimators and correlators," IEEE Trans. Inform. Theory, vol. IT-28, pp. 924-932, Nov. 1982.

[10] E. E. Azzouz and A. K. Nandi," Automatic modulation recognition of communication signals," Kluwer Acadamic Publisher 1996.

[11] A. W. Wegner, "Practical techniques for baud rate estimation," European Association For Signal Processing IV conf. 1992, pp. 681684.

[12] E.E.Azzouz, "baud duration estimation techniques," $14^{\text {th }}$ Radio science conf. March 1997, Cairo university, Egypt.

[13] A. Al-Makhlouf,"Analysis of the performance of EW systems: Parameters Estimation of Digitally Modulated Signals," Msc. thesis, MTC, Cairo, Nov. 1997. 
Proceedings of the $\mathbf{2}^{\text {nd }}$ ICEENG Conference, 23-25 Nov. 1999

\begin{tabular}{|l|l|}
\hline SP-7 & 9 \\
\hline
\end{tabular}

Table(1): Percentage of successful baud duration estimation of M-ary PSK signals based on 10 realizations and $2 \%$ estimation error.

\begin{tabular}{|c|c|c|}
\hline $\begin{array}{c}\text { SNR } \\
(\mathrm{dB})\end{array}$ & $\begin{array}{c}\text { BPSK } \\
( \pm 2 \%)\end{array}$ & $\begin{array}{c}\text { QPSK } \\
( \pm 2 \%)\end{array}$ \\
\hline 5 & 100 & 100 \\
\hline 0 & 100 & 90 \\
\hline-5 & 100 & 90 \\
\hline-7 & 100 & 90 \\
\hline
\end{tabular}

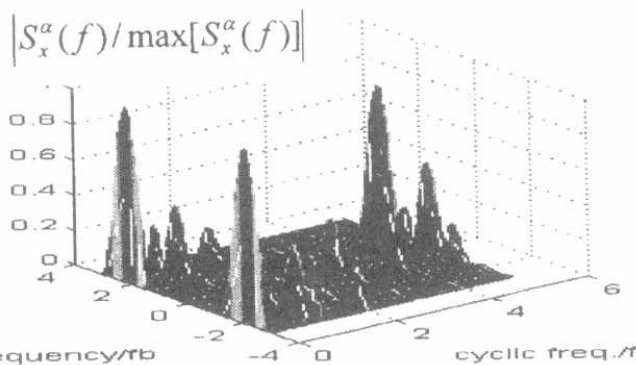

(a)

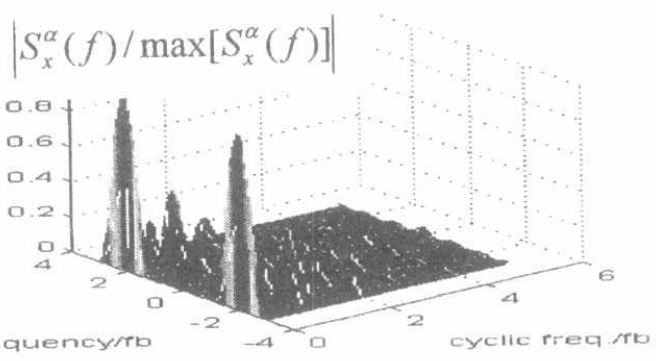

(b)

Fig. 1. Theoretical spectral correlation function magnitude for: (a) BPSK signal, and (b) QPSK signal

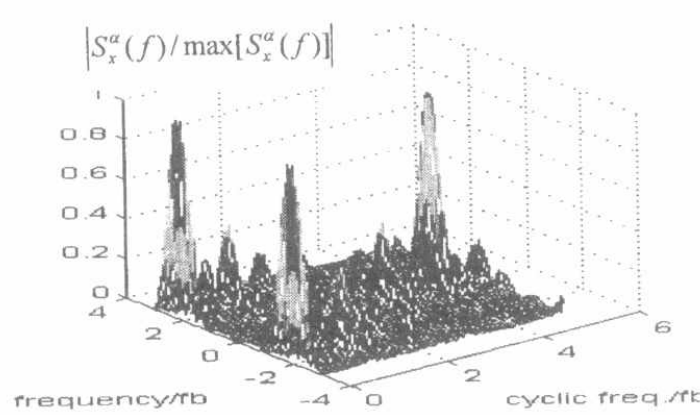

(a)

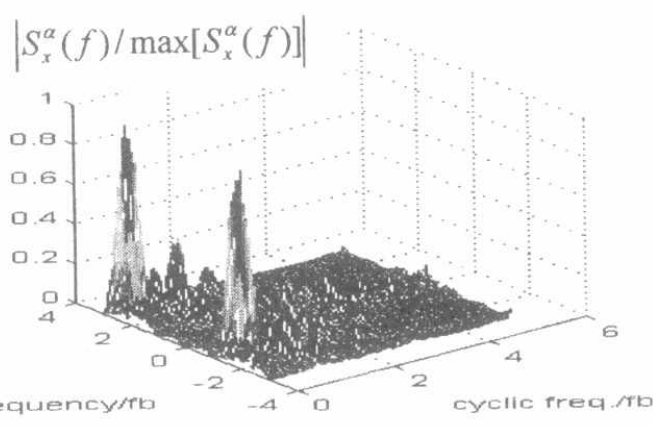

(b)

Fig. 2. Estimated spectral correlation function magnitude for: (a) BPSK signal, and (b) QPSK signal.

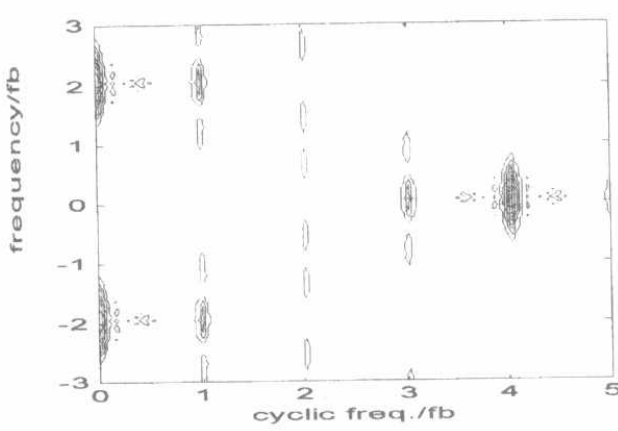

(a)

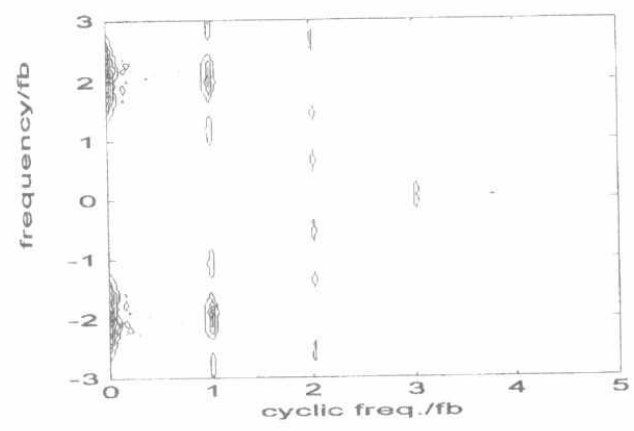

(b)

Fig. 3. Contours of estimated spectral correlation function magnitude for: (a) BPSK signal, (b) QPSK signal. 